livraisons

d'Histoire

de l'Architecture

\section{Livraisons de l'histoire de l'architecture}

$31 \mid 2016$

La source photographique dans la pratique de l'historien de l'architecture

\title{
La photographie critique au service d'une critique opératoire dans l'œuvre de Paolo Portoghesi
}

Critical photography used as operative critics in Paolo Portoghesi's work

Die kritische Fotografie im Dienste einer Verfahrenskritik im Werk von Paolo

Portoghesi

\section{Benjamin Chavardes}

\section{(2) OpenEdition}

Journals

Édition électronique

URL : http://journals.openedition.org/lha/584

DOI : $10.4000 /$ /ha. 584

ISSN : 1960-5994

Éditeur

Association Livraisons d'histoire de l'architecture - LHA

Édition imprimée

Date de publication : 14 juillet 2016

Pagination : 23-37

ISSN : $1627-4970$

Référence électronique

Benjamin Chavardes, «La photographie critique au service d'une critique opératoire dans l'œuvre de Paolo Portoghesi », Livraisons de l'histoire de l'architecture [En ligne], 31 | 2016, mis en ligne le 14 juillet 2018, consulté le 20 avril 2019. URL : http://journals.openedition.org//ha/584 ; DOI : 10.4000//ha.584

Ce document a été généré automatiquement le 20 avril 2019.

Tous droits réservés à l'Association LHA 


\title{
La photographie critique au service d'une critique opératoire dans l'œuvre de Paolo Portoghesi
}

\author{
Critical photography used as operative critics in Paolo Portoghesi's work \\ Die kritische Fotografie im Dienste einer Verfahrenskritik im Werk von Paolo \\ Portoghesi
}

Benjamin Chavardes

1 Paolo Portoghesi voit le jour en 1931, en plein cœur du centre historique de la Rome fasciste. Il grandit dans un climat d'instrumentalisation de l'histoire, en observant les transformations effectuées par le régime dans la capitale italienne. Au lendemain de la Seconde Guerre mondiale, il entreprend ses études à l'école de Rome, fondée par Gustavo Giovannoni (1873-1947). Il y est formé par un corps enseignant composé par les anciens architectes du régime, comme Arnaldo Foschini (1884-1968), auxquels on a assigné des assistants antifascistes comme Ludovico Quaroni (1911-1987). Portoghesi fait également partie de la première promotion à bénéficier de l'enseignement de Saverio Muratori (1910-1973). Il est alors influencé par les recherches identitaires de l'architecture italienne de l'après-guerre et notamment par les travaux «néoréalistes " de Mario Ridolfi. Il commence très tôt, parallèlement à ses études, à entreprendre des recherches historiques, nourri par les enseignements d'histoire de Vincenzo Fasolo (1885-1969) et du futur président de l'ICOMOS (1964), Guglielmo De Angelis d'Ossat (1907-1992).

2 Portoghesi est conscient de la valeur provisoire de l'analyse historique. Il se place dans la lignée de Siegfried Giedion lorsque celui-ci soutient que : «L'historien est comme tout le monde, l'homme d'une époque ${ }^{1}$. » Pour Paolo Portoghesi :

« Traductions, perceptions et jugements font partie du flux continu de l'histoire et ne peuvent en être soustraits sans perdre leur sens, une méthode historico-critique pour comprendre l'architecture et transmettre cette compréhension implique des temps et des modalités $[. . .]^{2}$.»

3 Il définit sa méthode comme « historico-critique ». Il utilise la métaphore littéraire pour apparenter le travail de l'historien à celui d'une lecture et d'une traduction, et le travail 
du critique et de l'interprétation à une relecture. Portoghesi distingue trois temps de l'étude. Le premier est celui de la «lecture». L'historien doit pouvoir se projeter dans l'édifice dans son état et son époque d'origine. Cela implique une érudition suffisante. Puis, durant une seconde phase dite "documentaire", le travail philologique doit permettre de retracer le parcours de l'architecte-auteur depuis la feuille blanche jusqu'à la réalisation. Cette ambition suppose que les versions provisoires et définitives aient été conservées (textes, esquisses, plans...). À ce stade, l'utilisation du dessin par l'historien permet de reconstruire la géométrie de l'objet architectural, à l'image des reconstructions géométriques qui ouvrent Borromini nella cultura europea (Rome, Officina, 1964). Enfin, vient le temps de l'interprétation, le «moment «noble» de l'histoire de l'architecture » selon Portoghesi. Cet exercice de « relecture » doit permettre de définir la valeur et la signification de l'œuvre au moment où elle fut conçue et réalisée et également au moment de l'analyse. Cette méthodologie associe à la fois la rigueur scientifique de l'étude des sources de De Angelis d'Ossat et l'étude de l'organisme architectural de Fasolo. Pour étudier la géométrie de l'espace bâti, Portoghesi utilise le dessin mais développe surtout l'utilisation de la photographie.

\section{Médium fondamental}

4 Portoghesi appartient à la génération qui a vu se démocratiser la photographie et apparaître la couleur. Dans ses ouvrages, il consacre une place importante à l'image et, en particulier, à la photographie. Des pages entières de ses ouvrages sont consacrées aux illustrations. Déjà dans son ouvrage sur Guarini, pour vingt-quatre pages de texte on compte soixante-quatre pages d'illustrations. Sur les quarante-deux photos, quarante sont de Portoghesi, et c'est ce qu'il met en avant dans son courrier à Mario Labò ${ }^{3}$. Roma barocca comporte quatre cent soixante-quinze photos pour un volume de cinq cent deux pages. Les pages de photos représentent la moitié de l'ouvrage. Les publications de Portoghesi sont de beaux et grands formats permettant de mettre en avant les photos. Ce n'est pas le texte qu'il cherche à souligner par ce choix mais bien l'image. La photographie occupe donc une place importante dans le travail de l'historien. Le travail photographique de Portoghesi n'a pas manqué d'être remarqué voire d'être utilisé. Dès 1953, Benevolo se sert des photographies de Portoghesi pour illustrer son propos ${ }^{4}$. En 1957, Leonardo Sinisgalli évoque la «[...] documentation photographique merveilleuse de ses explorations borrominiennes ${ }^{5}$. " Dans sa critique, pour le moins acerbe, sur Bernardo Vittone: un architetto tra Illuminismo e Rococo, Richard Pommer débute par l'attention portée au travail photographique :

«Il a utilisé majoritairement ses propres images ; certaines avec un objectif Nikkor "fish-eye", d'autres avec un instantané : fragmentaire, déformé, délavé, brouillard sentimental, poétique, décontracté, spectaculaire, souvent des enregistrements de l'appareil photo et non de l'édifice, mais là encore, pas Alinari. La surabondance donne l'illusion de regarder l'édifice, ou d'être là-bas avec notre $35 \mathrm{~mm}$ : ce qui est vrai de ce que nous faisons de l'économie d'illustration, est encore moins respecté par la pauvreté scientifique que par l'illusion de rigueur scientifique. Portoghesi n'est pas seulement généreux - 363 photos, souvent une dizaine pour un bâtiment et des dessins - mais il n'a pas peur d'exploiter le luxe du beau livre grand format, qui nous méprise pour quelques-unes des mêmes raisons snobs que le photographe : ses pages ont le luxe d'un photomontage, parfois d'un Rauschenberg, et c'est la prodigalité et la variété ou même l'éclectisme sauvage qui importe, et non tant la qualité d'un photographe indépendant : $35 \mathrm{~mm}$ contre 8 " x 10"6. » 
Henry A. Millon met l'accent sur la qualité des photos dans The Rome of Borromini, Architecture as Langage ${ }^{7}$. Léon Krier évoque également le travail photographique de Portoghesi sur Michel-Ange et les photographies de San Ignazio dans Roma barocca ${ }^{8}$. On peut également citer le commentaire de Rudolf Wittkower sur le Borromini de 1967 :

«Il fait ressortir une interprétation du langage architectural de Borromini, avec

l'aide de magnifiques photographies'. »

La photographie est un outil historico-critique remarquable dans la mesure où elle offre à considérer très précisément l'objet photographié, sélectionné par le regard du preneur de vue. En effet, on photographie ce que l'on voit, certes un témoignage précis de l'objet, mais on fige cependant un regard moderne, nouveau et personnel sur celui-ci. À l'image de la mission héliographique, ou du travail de Paolo Monti à Bologne ${ }^{10}$, l'utilisation de la photographie comme outil de mémoire n'est pas nouvelle. Dans son ouvrage, Roma un'altra città, le travail photographique que réalise Portoghesi pour Rome consiste à dépeindre la ville d'avant les travaux de l'époque fasciste, tel un archéologue, avec des images choisies, en cherchant à communiquer des ambiances passées. Il s'agit d'un travail qui vise à définir une identité à travers les images sélectionnées. La photographie, contrairement à la peinture, dépeint une réalité spontanée avec les relations volumétriques, les spatialités, la luminosité et les matières. L'ouvrage comporte seulement trente-quatre pages de texte pour trois cent quarante-trois pages de photos. Comme le souligne Giovanni Fanelli, Portoghesi «[...] utilise ses photographies comme puissant moyen d'investigation, de lecture et d'interprétation formelle passionnée des caractéristiques spécifiques des œuvres analysées ${ }^{11}$.»

\section{Photographie critique}

7 Manfredo Tafuri évoque ce travail de " photographie critique ${ }^{12}$ » et le risque qu'il génère de réaliser des images autonomes n'ayant plus de lien avec l'objet étudié. Trois noms sont cités en exemples : Bruno Zevi, Leonardo Benevolo et Paolo Portoghesi. La photographie de l'une des nefs latérales de Saint-Jean de Latran par Portoghesi dans l'ouvrage sur Borromini (1967) illustre très bien le propos de Tafuri ${ }^{13}$ : la colonnade est photographiée comme une composition abstraite du début du $\mathrm{XX}^{\mathrm{e}}$ siècle. Seul le coin inférieur gauche laisse apparaitre les bases des colonnes permettant ainsi de l'identifier. Le fait que cette image occupe une double page permet de penser que ce qui est une critique chez Tafuri, est une démarche assumée chez Portoghesi. La colonnade de Saint-Jean de Latran n'est qu'un exemple parmi d'autres de photographies de détails architecturaux où l'abstraction esthétique prend le pas sur le caractère documentaire. Mais, l'abstraction devient aussi un médium pour une utilisation contemporaine d'une lecture historique dans le projet (ill. 1). 
III. 1 : Nef latérale de la basilique Saint-Jean-de-Latran de Francesco Borromini

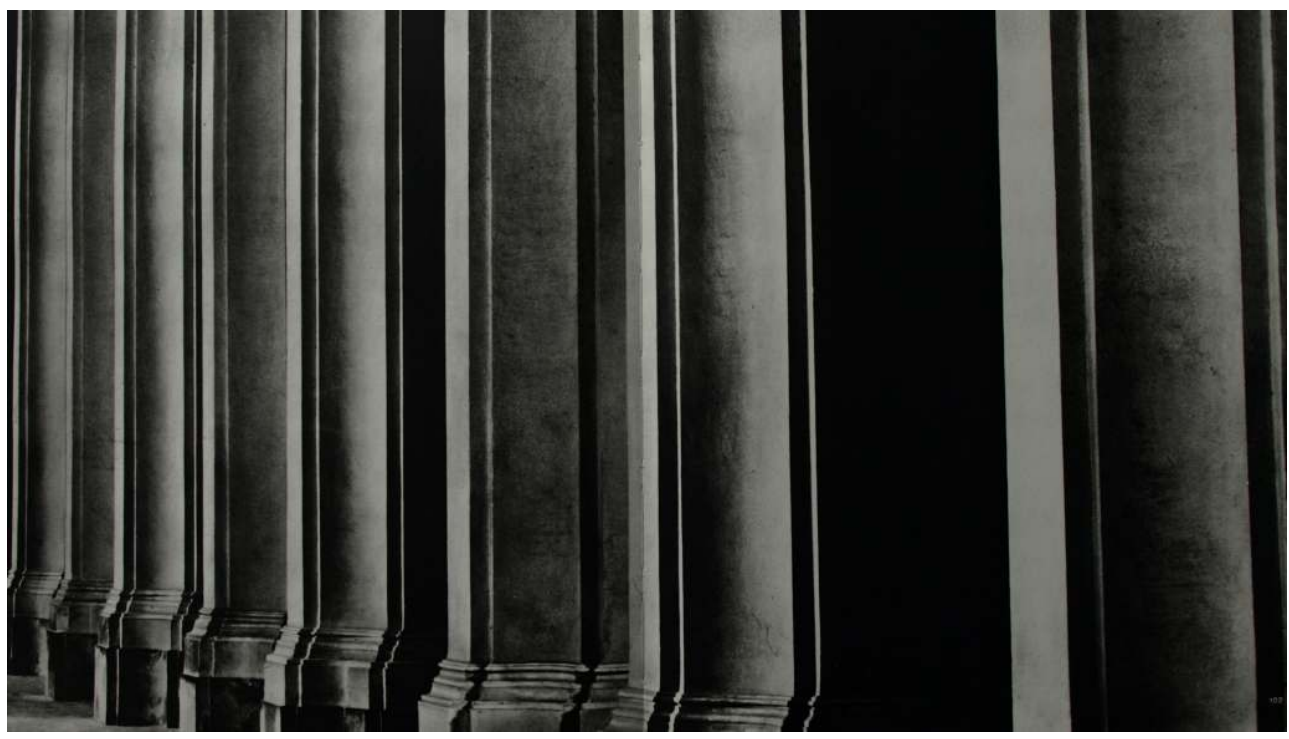

Borromini. Architettura come linguaggio, Milan, Electa, 1967. Trad. fr. : Borromini, Architecture, Langage, Paris, Vincent, Fréal \& Cie, 1969, p. 101-102.

(c) Cl. Paolo Portoghesi

8 La photographie de détail trouve également une utilisation dans la comparaison formelle entre deux époques. Cette méthode fait référence à la pédagogie d'Heinrich Wölfflin (1864-1945) qui utilisait simultanément deux projecteurs dans ses cours ${ }^{14}$. L'ouvrage sur le baroque et l'Art nouveau de Portoghesi reprend cette méthodologie en confrontant sur deux pages en vis-à-vis deux édifices présentant des similitudes formelles, l'un baroque et l'autre Art nouveau ${ }^{15}$. L'utilisation régulière de photographies en contre-plongée est également particulièrement frappante. Que ce soit dans l'espace urbain ou architectural :

« On doit apprendre à regarder aussi en haut et en bas : en haut vers cette tranche

variable de ciel (parfois mince comme un couteau dans les rues de Naples ou Gênes)

qui conclut l'espace des rues ${ }^{16}$.»

Ces photographies mettent ainsi l'accent sur la géométrie des édifices à travers le tracé des corniches. En 1963, la société Nikon sort le premier objectif hypergone, plus communément appelé fisheye. Il présente la particularité d'avoir une distance focale très courte et donc un angle de champ très grand, jusqu'à cent quatre-vingt degrés dans la diagonale. Par conséquent, les lignes droites qui ne passent pas par le centre de l'image subissent une forte distorsion et sont courbées ${ }^{17}$. Dès 1966, Portoghesi utilise cet objectif dans ses ouvrages, comme le souligne Pommer ${ }^{18}$. Ces vues sont en réalité la version photographique des perspectives axonométriques plafonnantes d'Auguste Choisy, appréciées de Gustavo Giovannoni et intégrées à l'enseignement de Vincenzo Fasolo (ill. 2). 
III. 2 : Plafond de l'église San Carlino alle Quattro Fontane de Francesco Borromini

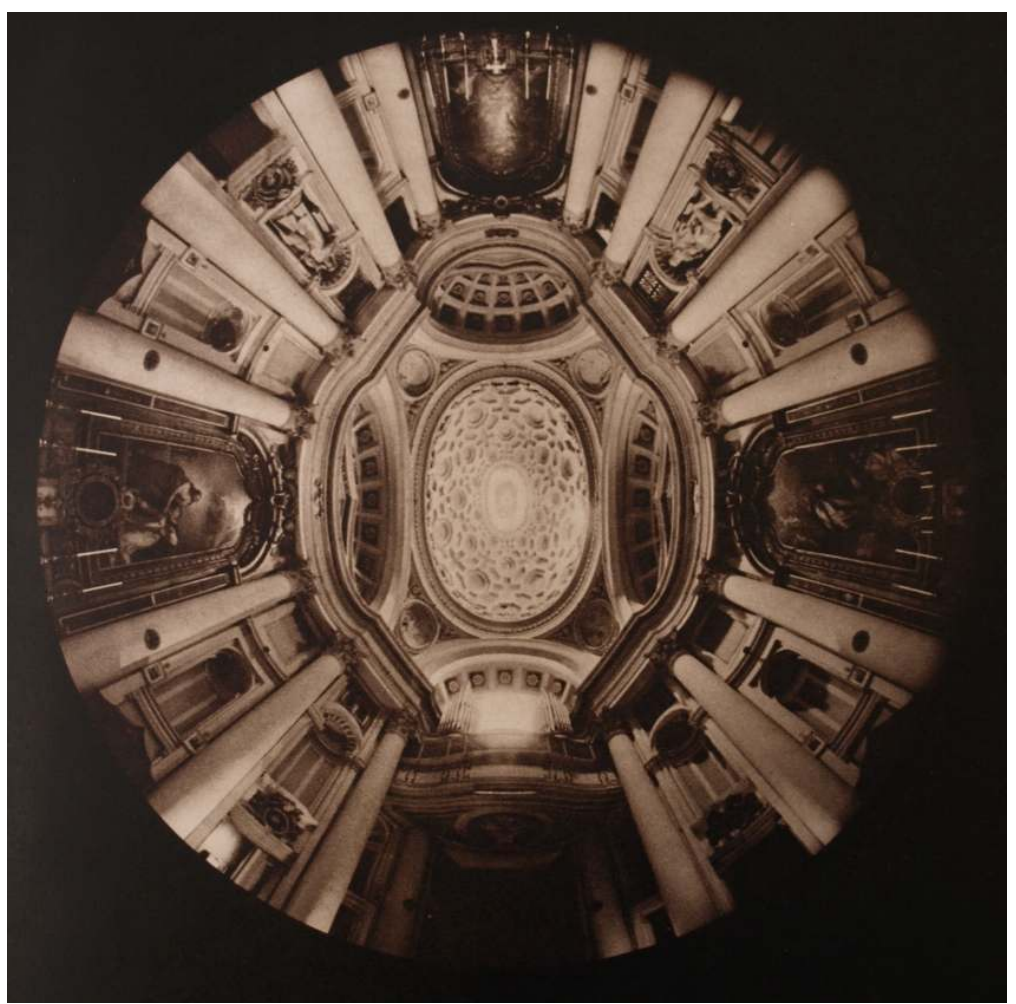

Borromini nella cultura europea, Rome, Officina, 1964.

(C) Cl. Paolo Portoghesi

En septembre 2011, Paolo Portoghesi propose une visite guidée de San Carlo alle Quattro Fontane dans le cadre du troisième cours international organisé par le Centro internazionale di studi «Jacopo Barozzi da Vignola ». Il connaît bien cet édifice dont il a exploré les moindres recoins depuis plus de soixante ans jusqu'à publier la monographie la plus complète à ce jour sur le sujet ${ }^{19}$. Alors que le groupe explore la crypte, Portoghesi sort de sa poche un petit appareil photo ${ }^{20}$. Nous avons pu constater qu'il se déplace toujours avec au moins un petit appareil photo sur lui, ce qui démontre l'importance qu'il attache à cet instrument. Il explique que c'est un outil de mesure pour l'architecte ${ }^{21}$.

\section{Critique opératoire}

11 Portoghesi souligne à quel point le fait d'être architecte praticien apporte une vision plus concrète à l'historien :

«Un des petits avantages qu'offre le fait d'être à la fois critique et architecte praticien est de savoir à quel point les sources et les documents sont susceptibles, à quelques époques que ce soient, de falsifications et d'erreurs involontaires, combien la vérité historique peut être mise sous le boisseau par des facteurs contingents qui influencent l'enregistrement documentaire ${ }^{22}$."

Giulio Carlo Argan définit ainsi la méthode historique de Paolo Portoghesi :

«La méthode historique de Portoghesi consiste non pas à retrouver Palladio chez Aalto ou Borromini chez Wright, opération relativement facile mais, à l'inverse, à trouver Aalto chez Palladio et Wright chez Borromini, ce qui est beaucoup plus difficile; donc, à démontrer que Palladio et Borromini étant ce qu'ils sont, Aalto, 
Wright et ce qui suit, y compris l'engagement moral personnel de l'historien, ne pouvaient pas ne pas exister. On entre ainsi dans un ordre de nécessité qui veut que l'historien ne peut exister qu'en tant qu'être politique: la poétique n'est pas la prémisse mais la nécessité éthique de l'engagement sur le plan opératoire de l'art ${ }^{23}$

.»

13 Comme le décrit Argan, la recherche historique de Portoghesi tend à démontrer que ce qui a suivi était inéluctable et que cela été prévisible au vu de ce qui fut. Portoghesi cherche non tant l'héritage que la prévision. Il est donc dans une recherche de la continuité et de la genèse.

Portoghesi défend l'importance d'entretenir un lien avec le passé comme un critère pour s'inscrire dans la continuité historique et dans la continuité du mouvement moderne. On retrouve l'esprit qui anime tout ou partie de la scène architecturale de l'après-guerre en Italie et notamment la continuité critique portée par Ernesto Nathan Rogers et la revue Casabella continuità.

La stratégie consistant à recourir à l'histoire pour légitimer un positionnement théorique est classique et représentative de la démarche de Portoghesi. Son argumentation ne repose pas sur une analyse de la situation présente, mais sur une continuité des valeurs et des fonctionnements en faisant appel à l'histoire, à la tradition, ou bien encore à la mémoire. Le récit de Portoghesi fonctionne donc comme un hypertexte mobilisant une érudition suffisante pour retisser les liens entre les différentes références mobilisées. Mais au-delà, l'intention de Portoghesi est de réutiliser l'histoire dans le processus de conception :

«L'engagement de ma recherche, que ce soit dans le champ de l'architecture aussi bien que dans l'histoire de l'art, a été celui d'affirmer une idée de connaissance historique qui, sans rien sacrifier des conquêtes critiques de la culture moderne, puisse rapporter les livres d'histoire sur la table à dessin $[\ldots]^{24}$.»

Il poursuit ainsi une recherche qu'il a partagée un temps avec Bruno Zevi (1918-2000), lorsqu'ils montèrent ensemble l'exposition sur Michel-Ange de 1964. La volonté est de définir une matrice en récupérant les processus qui président à la naissance d'une forme historique.

\section{Michel-Ange architecte (1964)}

17 À l'occasion des célébrations des 400 ans de la mort de Michel-Ange (1475-1564), une exposition est organisée sur l'œuvre du maitre au Palazzo delle Esposizioni dans la via Nazionale à Rome. Elle est l'illustration du travail de critique opératoire mené par Paolo Portoghesi et Bruno Zevi.

Dans son ouvrage, publié en 1948, Saper vedere l'architettura, Zevi définit l'espace intérieur comme la caractéristique intrinsèque de l'architecture ${ }^{25}$. De ce point de vue, le sous-titre est significatif: Saggio sull'interpretazione spaziale dell'architettura. La scénographie et le matériel utilisé pour l'exposition illustrent cette volonté d'explorer le regard porté sur l'architecture. Les photographies sont orientées de diverses manières, accrochées à divers niveaux depuis le sol. Comme l'écrit Zevi, en introduction du volume sur Michel-Ange : "Ce livre est le produit indirect d'un travail universitaire réalisé à Venise durant trois années, de l'automne 1960 à l'été $1963^{26}$.» Les travaux des étudiants de l'IUAV sont amplement utilisés: dessins critiques, photographie créative et maquettes interprétatives. On trouve ainsi les maquettes interprétatives de la Nouvelle Sacristie de 
la basilique San Lorenzo à Florence, des projets de fortifications de Florence, de la bibliothèque Laurentienne, de la place du Capitole, de la basilique Saint-Pierre de Rome, de la chapelle Sforza à Santa Maria Maggiore et de Santa Maria degli Angeli. Les dessins et les maquettes sont donc utilisés à la fois comme outils d'enseignement et comme outils d'analyse critique. Les matériaux utilisés varient selon les éléments mis en évidence ; ils mettent en exergue les lignes de forces, les formes, la volumétrie et les dynamiques spatiales d'un édifice. Les interprétations spatiales deviennent suffisamment abstraites pour permettre d'envisager une réutilisation contemporaine. C'est le sens de cette critique opératoire, où l'abstraction devient le medium entre étude historique et la pratique du projet (ill. 3).

\section{3 : Exposition monographique Michelangiolo architetto}

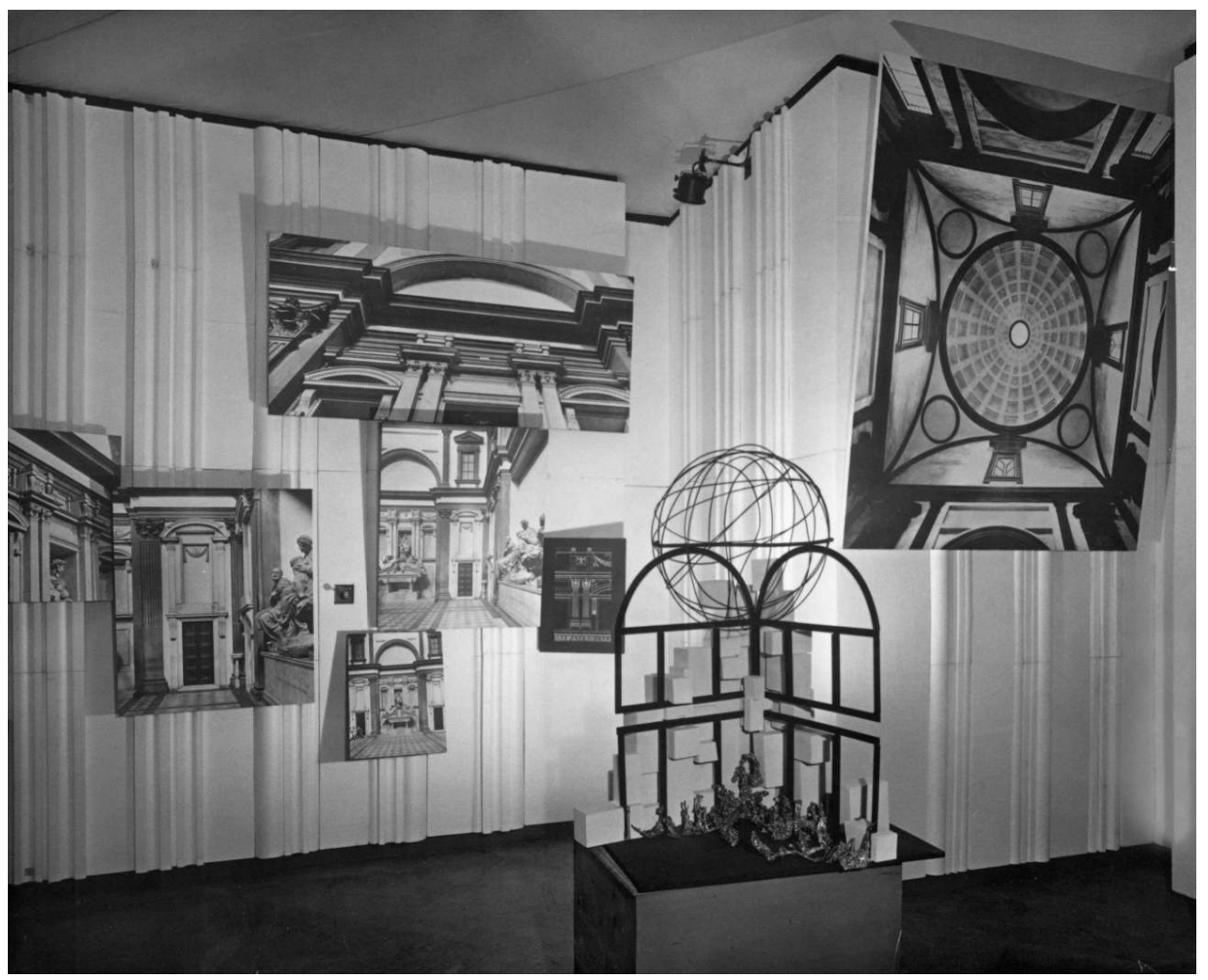

Palazzo delle esposizioni, Rome, 1964.

(c) Cl. Paolo Portoghesi

La scénographie de l'exposition constitue la première collaboration de Portoghesi avec l'ingénieur Vittorio Gigliotti. En 1961, ce dernier crée le Studio AZ Architetti e ingeniori avec Zevi. Portoghesi transforme complètement l'espace intérieur du Palazzo delle esposizioni. Entre les partitions existantes de l'édifice, il réalise un parcours de visite qui, sur le plan, prend la physionomie "d'une sorte de grand appareil digestif ${ }^{27}$ ", pour reprendre ses propres mots. Les parois sont réalisées avec des panneaux de polystyrène blancs. Différents modules sont assemblés dans une grande variété de configurations, afin de délimiter l'espace d'exposition. Le plan et les modénatures réinterprètent de façon plus ou moins abstraite les formes architecturales des œuvres de Michel-Ange. Le plan de chaque salle est réalisé en écho à l'œuvre présenté dans celle-ci. Ainsi, la salle consacrée au projet de tombeau pour Jules II a une forme ovale comme le plan intérieur du premier projet de Michel-Ange (1505-1506). Dans la continuité chronologique et spatiale de 
l'exposition, la salle sur la nouvelle sacristie de San Lorenzo (1519-1534) a également un plan carré avec des angles dont les modénatures sont accentuées. Puis, Portoghesi reprend la configuration en coude de l'entrée de la bibliothèque Laurenziana (1519-1559), suivi de l'espace longitudinal de la bibliothèque. La salle de projection reprend quant à elle les dessins de Michel-Ange pour les fortifications de Florence (1528-1529). De même, il reprend le plan du projet de la chapelle Sforza à Sainte-Marie Majeure en plan. Le travail sur les parois est mis en valeur par un décollement de celles-ci par rapport au sol et au plafond d'une part et par le contraste entre le blanc des parois et l'aspect sombre des sols et des plafonds. Tout au long du parcours, une attention particulière est portée aux modénatures dans l'ensemble des panneaux de polystyrène et notamment vers la fin du parcours, où est conçu une rampe des modénatures, sorte de répertoire formel qui dessine les parois du cheminement. L'aménagement de Portoghesi est donc directement lié à Michel-Ange, dans la construction de l'espace intérieur et dans la modénature des façades intérieures, mais généré par une réinterprétation abstraite libre afin de créer une atmosphère originale mais qui fait écho à l'architecture du maitre maniériste (ill. 4).

\section{4 : Plan de l'exposition monographique Michelangiolo architetto}

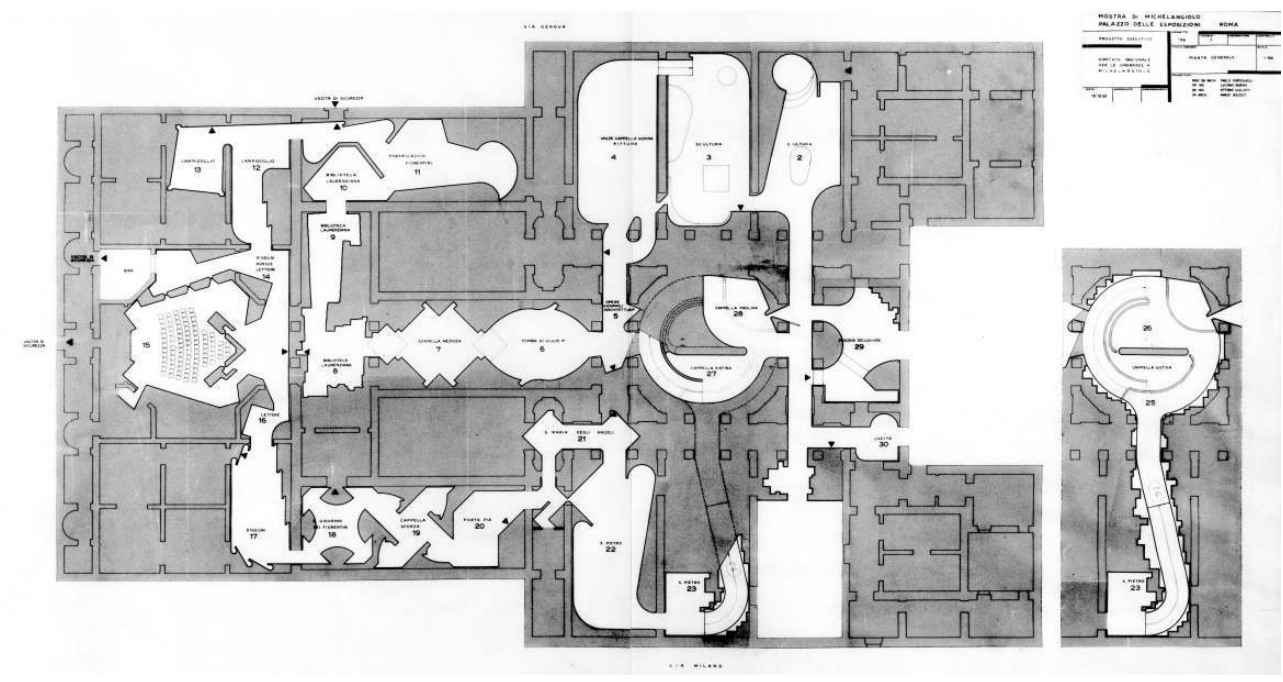

Palazzo delle esposizioni, Rome, 1964.

(C) Cl. Paolo Portoghesi

\section{Zevi, Portoghesi et la photographie}

Après l'ouvrage sur Michel-Ange, Zevi et Portoghesi décident de collaborer sur un ouvrage consacré à Borromini. Dans un courrier daté du 27 décembre 1966, Bruno Zevi propose également à Paolo Portoghesi et Manfredo Tafuri un "projet historique " comportant quatre parties : une histoire de l'architecture en Italie, une histoire générale de l'architecture, une encyclopédie de l'architecture et une collection d'histoire de l'architecture. Il développe plus précisément la première partie. La proposition prend la forme d'un texte introductif de présentation et d'une déclaration commune à amender ${ }^{28}$. Ces projets ne verront pas le jour, ou du moins pas nécessairement sous la plume de ce trio $^{29}$. L'ouvrage sur Borromini fut complètement rédigé mais jamais publié. Zevi s'y oppose lorsqu'il découvre la monographie publiée en 1967 par Portoghesi chez Electa. Cette année est marquée par les célébrations du tricentenaire de la mort de Francesco 
Borromini (1599-1667). À cette occasion, plusieurs évènements sont organisés et les publications se multiplient. Portoghesi signe le catalogue de l'exposition à l'Accademia Nazionale di San Luca ${ }^{30}$. Une autre exposition, présentant les dessins et les documents propriétés du Saint-Siège, est organisée au Vatican avec un catalogue signé par $\mathrm{H}$. Thelen ${ }^{31}$. Un colloque réunit en plus de Christian Norberg-Schulz, quelques-uns des historiens de l'architecture italiens les plus reconnus: Giulio Carlo Argan, Eugenio Battisti, Marcello Fagiollo, Paolo Marconi, Luigi Moretti, Manfredo Tafuri et enfin Bruno Zevi et Paolo Portoghesi ${ }^{32}$. Ces deux derniers vont se livrer une "passe d'armes" sur le thème de l'actualité de Borromini et l'influence que peut exercer Borromini sur les architectes maitres d'œuvre en 1967. La polémique est ouverte par la communication de Bruno Zevi qui dénonce ceux qui voudraient «berninizzare Borromini », c'est à dire qui voudraient s'attirer les faveurs de Borromini, en trahissant la réalité du personnage. Pour argumenter sa démonstration, il définit cinq procédés utilisés, qui démontrent clairement que son "attaque " vise spécifiquement Portoghesi. Le troisième reproche dénonce l'aspect hypnotique des photographies. C'est le reproche envers Portoghesi le plus explicite :

«Durant le cours monographique sur Borromini, j'ai projeté de nombreuses photographies préparées, sous la direction habile de Paolo Portoghesi, pour le volume de Einaudi, qui sont pour beaucoup des photos montrées dans les expositions. Les élèves ont été enchantés, séduits, persuadés. Puis, presque chaque semaine, nous sommes allés visiter une œuvre de Borromini. Quand ils la voyaient, découvrant les véritables espaces et volumes, ils ont été déçus, il était difficile de déchirer l'état hypnotique créé à partir des photographies et les conduire à rencontrer la vraie architecture, qui n'est pas du tout convaincante et rassurante, mais choquante, dramatique, tragique ${ }^{33}$. »

Ce débat laissera des marques profondes et scelle la rupture entre les deux protagonistes.

\section{Photographie critique et projet architectural}

La comparaison entre les œuvres bâtis de Portoghesi et les photographies de ses études historiques permettent de retrouver les processus engagés. Ainsi, les gradins d'ombres et de lumière des colonnes de la nef de Saint-Jean-de-Latran se retrouvent dans le sfumato du plafond de l'église de la Sacra Famiglia à Salerne ${ }^{34}$.

Portoghesi établit lui-même un lien formel entre le temple rond de Baalbek et la casa Baldi dans la préface qu'il rédige en 1964 pour l'ouvrage de Gilbert Picard sur l'Empire romain ${ }^{35}$ - On peut réaliser cet exercice de comparaison formelle dans d'autres œuvres de Portoghesi, comme les coupoles de l'église Saint-Charles-des-Quatre-Fontaines de Borromini et de la mosquée de Rome, ou bien les superpositions de structure de la chapelle de Vallinotto de Bernardo Vittone, l'église San Lorenzo de Guarino Guarini à Turin et la mosquée de Rome, ou bien encore, les angles ouverts de l'oratorio dei Filipini de Borromini et ceux de l'église de Salernev (ill. 5). 

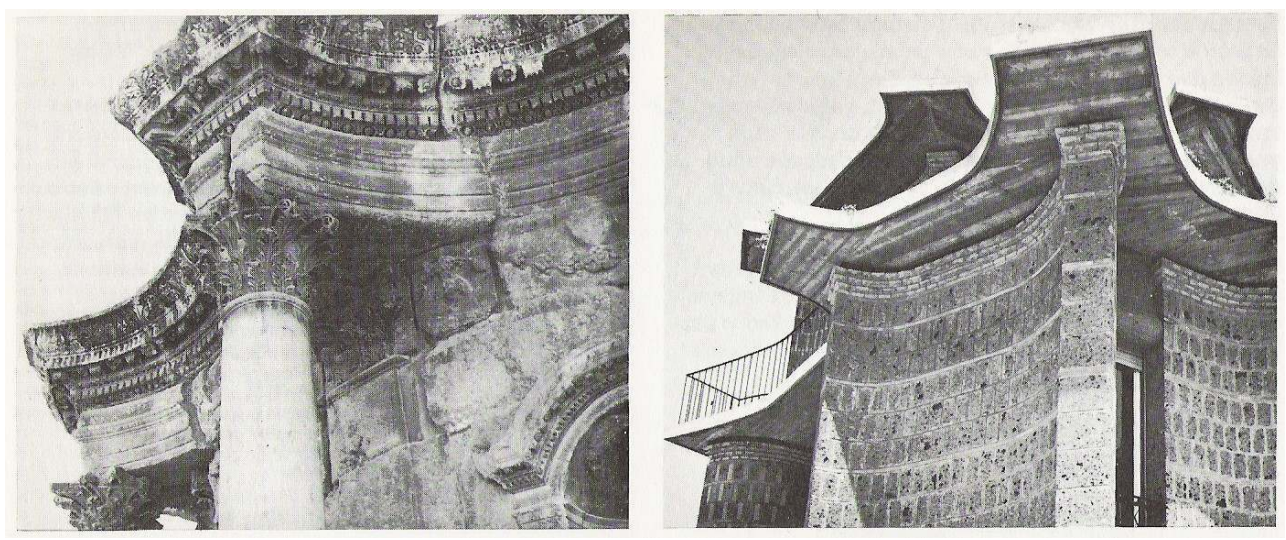

Par Paolo Portoghesi, dans « Rome et l'architecture organique », préface à Gilbert Picard, Empire romain, Fribourg, Office du Livre, 1965, p. 6.

(C) Cl. Paolo Portoghesi

Portoghesi utilise également son objectif Fisheye pour photographier ses propres œuvres et mettre ainsi l'accent sur la géométrie qui privilégie alors la centralité de l'organisme architectural.

Pour l'exposition Roma Interrotta (1978), son arpentage de la campagne du Latium a eu pour objectif de comprendre et de définir ce qui constitue pour lui les «lignes structurelles du paysage ${ }^{36}$ » et d'en extraire des liens avec son exploration de la ville de Rome. En faisant des rapprochements formels entre les deux par le biais, entre autres, de la photographie, il a eu l'ambition d'établir des continuités territoriales. L'idée directrice du projet est que, si la forme urbaine obéit aux mêmes règles de composition que le paysage, alors il est possible de générer une continuité entre les deux.

Ainsi, plusieurs planches établissent les similitudes entre le paysage naturel du Latium et celui urbain de Rome par le biais de photographies comparées. Il met en évidence des analogies entre tracé urbain et tracé des ravins. Il reconstruit ainsi une hypothèse de l'environnement physique originel de Rome, c'est à dire une « Roma prima di Roma », avant que les inondations et les éboulements ne modèlent un autre paysage, aplatissant la physionomie originelle du terrain. De cette manière, il réintègre la nature en ville non plus dans des zones circonscrites ponctuelles mais dans la physionomie même de la cité (ill.6). 


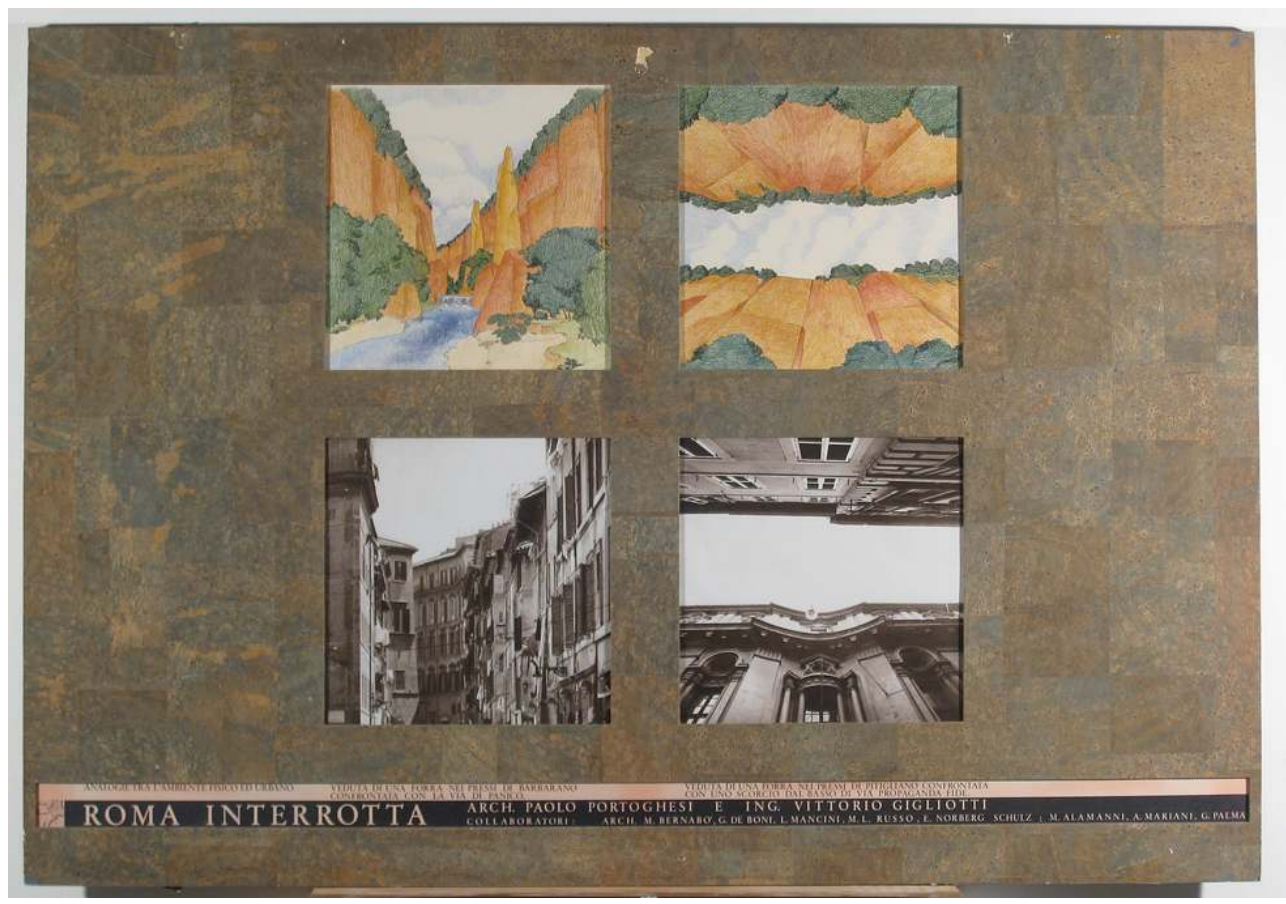

Contribution de Paolo Portoghesi à l'exposition Roma interrotta, Rome, 1978.

(C) $\mathrm{Cl}$. Paolo Portoghesi

\section{Les valeurs libres}

Comme le souligne Portoghesi: "Dans l'histoire de l'architecture les instruments de contrôle indirect ont toujours existé ${ }^{37}$. " Il s'inscrit ainsi dans une longue tradition qu'ont les architectes de vouloir établir des règles et des méthodes de composition. Il cite ainsi la méthode additive tardogothique, le rapport entre masse et espace dans les projets de Michel-Ange pour les fortifications de Florence et la contraction et la dilatation de l'espace perceptif chez Borromini. Il reconstruit dans ses études historiques les tracés géométriques des œuvres de Guarini et surtout de Borromini. Il révèle ainsi les lois géométriques qui gouvernent la composition. Ces schémas interprétatifs deviennent la matrice d'une nouvelle recherche. Dans la première partie de sa carrière la perspective est le point de départ de ses études mais le travail en plan est l'instrument déterminant. Il y a un départ instinctif qui trouve une loi géométrique ${ }^{38}$.

Ce travail géométrique est à la fois la conséquence et le complément de ce qu'il nomme les valeurs libres :

« La reprise, dans un système unique de l'histoire de l'architecture en général, et de l'histoire de l'architecture moderne, met en évidence automatiquement une série de valeurs laissées libres dans le cours du développement historique, d'hypothèses avancées mais non développées, de recherches interrompues au seuil de réalisations extraordinaires, de richesses et de potentialités gaspillées. De nombreux aspects de mon expérience d'architecte sont liées à la découverte de ces valeurs libres, à la tentative de les récupérer dans un nouveau contexte en continuant la recherche là où elle s'était interrompue, là où elle se révèle annonciatrice de nouvelle possibilités technologiques, de nouvelles possibilités d'utilisation, de nouvelles conditions historiques et sociales ${ }^{39}$. " 
Ainsi, il définit dans l'histoire des expériences inachevées qu'il réinvestit pour développer de nouvelles architectures. Ce travail se fonde sur des références historiques qui, à l'image de son écriture, génèrent une architecture hypertextuelle.

\section{Conclusion}

Les recherches des architectes italiens au lendemain de la Seconde Guerre ne peuvent être appréhendées en dehors d'une contextualisation spécifique. On ne peut porter un jugement historiciste sur l'architecture italienne sans mesurer les problématiques identitaires. Que ce soit à travers son parcours, ses positionnements ou ses travaux, Portoghesi est représentatif d'une époque et un acteur majeur du débat architectural et notamment de la question d'une recherche sur le lien entre histoire et projet architectural.

31 Paolo Portoghesi peut être considéré comme le principal représentant de la critique opératoire en ce sens que, contrairement à Zevi, il a construit. Beaucoup préfèrent aujourd'hui considérer Portoghesi comme un historien et non comme un architecte. Cette position s'explique d'une part par l'intérêt suscité par les publications de Portoghesi et de l'autre par un point de vue très critique sur son œuvre architecturale, laquelle demeure par ailleurs largement méconnue à l'exception de la casa Baldi et de la mosquée de Rome ${ }^{40}$. En réalité, Portoghesi fait de l'histoire comme un architecte et non pas nécessairement comme un historien. Le lien entre histoire et architecture est ténu et l'on ne peut pas comprendre son œuvre d'historien si l'on ne la repositionne pas dans une démarche préalable à l'acte de projet. Réciproquement, son œuvre architecturale n'est pas compréhensible si l'on ignore ses études historiques. Les deux sont intrinsèquement liées et forment une seule et même démarche. Il est évident qu'au-delà d'une pratique de l'histoire, ce qui importe dans sa démarche c'est la mise en œuvre d'une histoire pratique, autrement dit opératoire.

\section{NOTES}

1. Siegfried Giedion, Space, Time and Architecture: The Growth of a New Tradition, Boston, Harvard University Press, 1941. Trad. fr. d'Irmeline Lebeer et François-Maris Rosset, Espace, Temps, Architecture. La naissance d'une nouvelle tradition, Bruxelles, La Connaissance, 1968, p. 30.

2. Paolo Portoghesi, "Traduire une oeuvre architecturale", Les Cahiers de la recherche architecturale et urbaine, $\mathrm{n}^{\circ}$ 9/10, 2002, p. 14.

3. Le génois Mario Labò (1884-1961) est alors l'auteur de l'article sur Guarino Guarini dans l' Enciclopedia Italiana qui apparaît dans la bibliographie de l'ouvrage de Portoghesi.

4. Leonardo Benevolo, «Il tema geometrico di S. Ivo alla Sapienza », Quaderni dell'Istituto di Storia dell'Architettura, $n^{\circ}$ 3, novembre 1953, p. 1-10. En page 9, Benevolo utilise deux photographies de Paolo Portoghesi.

5. Leonardo Sinisgalli, «Esplorazioni borrominiane », La Fiera Letteraria, Rome, p. 1. Traduction de l'auteur. 
6. Richard Pommer, «Bernardo Vittone, Un architetto tra Illuminismo e Rococo by Paolo Portoghesi », The Art Bulletin, vol. 53, n 1, mars 1971, p. 124-125. Traduction de l'auteur.

7. Henry A. Millon, «The Rome of Borromini, Architecture as Langage by Paolo Portoghesi », Journal of the Society of Architectural Historians, vol. 31, n 3, octobre 1972, p. 243-245.

8. Commentaire de Léon Krier en marge de l'entretien réalisé par l'auteur le 27 février 2011.

9. Rudolf Wittkower, Art and Architecture in Italy : 1600 to 1750, Harmondsworth, Pelican Books, 1958. Trad. it. de Laura Monarca Nardini et Maria Vittoria Malvano, Arte e architettura in Italia 1600-1750, Turin, Einaudi, 1993, p. 467. Trad. fr. de l'auteur.

10. Italo Zannier, "Paolo Monti et le projet de Bologne ", Strates, $n^{\circ} 13$, Paysage urbain: genèse, représentations, enjeux contemporains [en ligne], mis en ligne le 22 octobre 2008, consulté le 15 août 2014, URL : http://strates.revues.org/6162.

11. Giovanni Fanelli, Storia della fotografia di architettura, Rome, Bari, Laterza, 2009, p. 362. Trad. fr. de l'auteur.

12. Manfredo Tafuri, Teoria e storia dell'architettura, Bari, Laterza, 1968. Trad. fr.: Théories et histoire de l'architecture, Paris, Société des architctes diplômés par le gouvernment (SADG), 1976, p. 215-217.

13. Paolo Portoghesi, Borromini. Architettura come linguaggio, Milan, Electa, 1967. Trad. fr., Borromini, Architecture, Langage, Paris, Vincent, Fréal \& C $C^{\mathrm{ie}}$, 1969, illustration 102.

14. Cette méthode est reprise par Giedion, qui la nomme Methodengleiche, et que connaîtra Christian Norberg-Schulz en tant qu'élève de ce dernier. On peut supposer que c'est peut-être par l'intermédiaire de son ami norvégien, que Portoghesi a été amené à réutiliser cette méthode. Jorge Otero-Pailos, Architecture's Historical Turn. Phenomenology and the Rise of the Postmodern, Minneapolis-Londres, University of Minnesota Press, 2010, p. 149.

15. Paolo Portoghesi, Luca Quattrocchi, Folco Quilici, Barocco e liberty, Lo specchio della metamorfosi, Gardolo, Luigi Reverdito, 1986. Trad. fr. de Anne Guglielmetti, Baroque et art nouveau, Le miroir de la métamorphose, Paris, Seghers, 1988, 207 p.

16. Paolo Portoghesi, Leggere e capire l'architettura, Rome, Newton \& Compton, 2006, 398 p., p. 8. Trad. de l'auteur.

17. L'utilisation de cet objectif a notamment été popularisée par son utilisation sur la pochette de l'album Big Hits High Tide and Green des Rolling Stones, sorti en 1966, et par les vues de l'ordinateur HAL9000 dans le film de Stanley Kubrick, 2001, l'Odyssée de l'espace, sorti en 1968.

18. Richard Pommer, "Bernardo Vittone... », op. cit.

19. Paolo Portoghesi, Storia di San Carlino alle Quattro Fontane, Rome, Newton \& Compton, 2001, $227 \mathrm{p}$.

20. «III Corso Internazionale di Studi sul Vignola: Vignola e la sua eredità tra Cinque e Seicento ", 12 au 17 septembre 2011. Cette anecdote a été relevée par l'auteur qui a suivi ce cours. 21. Entretien avec Paolo Portoghesi réalisé par l'auteur le 21 juin 2010.

22. Paolo Portoghesi, «Traduire une œuvre architecturale », op. cit.

23. Ibid., p. 18.

24. Paolo Portoghesi, Le Inibizioni dell'architettura moderna, Bari, Laterza, 1974, 248 p., p. 74. Traduction de l'auteur.

25. «L'espace interne, cet espace qui, comme nous le verrons, ne peut être complètement représenté d'aucune manière, qui ne peut être appris ni " vécu », sinon par l'expérience directe, est l'élément fondamental du fait architectonique. » Bruno Zevi, Saper vedere l'architettura, Turin, Einaudi, 1948. Trad. fr. de Lucien Trichaud, Apprendre à voir l'architecture, Paris, Minuit, 1959, 134 p., p. 11.

26. Paolo Portoghesi, Bruno Zevi, Michelangiolo architetto, Turin, Einaudi, 1964, 1019 p., p. 11.

27. Paolo Portoghesi, Le Inibizioni dell'architettura moderna, op. cit., p. 13.

28. Courrier de Bruno Zevi adressé à Paolo Portoghesi et Manfredo Tafuri, daté du 27 décembre 1966, archives de Paolo Portoghesi. 
29. La Storia dell'architettura italiana a vu le jour chez Electa. Zevi signe une histoire générale de l'architecture. Portoghesi dirige le Dizionario Enciclopedico di Architettura e Urbanistica (DAU). Pier Luigi Nervi dirige la collection d'histoire de l'architecture éditée par Electa.

30. Paolo Portoghesi, Disegni di Francesco Borromini, Mostra organizzata dall'Accademia Nazionale di S. Luca in occasione del terzo centenario della morte di Francesco Borromini, Rome, De Luca, 1967, 33 p.

31. Heinrich Thelen, Francesco Borromini die Handzeichnungen, Graz, Akademische Druck, 1967, n. p.

32. Colloque organisé par l'Accademia nazionale di San Luca à Rome du 28 septembre au 7 octobre 1967.

33. Bruno Zevi, «Attualità del Borromini ", Convegno di studi borrominiani, Palazzo Carpegna, Rome, Accademia Nazionale di San Luca, 1967, 547 p., p. 523. Trad. fr. de l'auteur.

34. Benjamin Chavardes, «De Francesco Borromini à Paolo Portoghesi. L'église de la Sacra Famiglia à Salerne ", Livraisons d'histoire de l'architecture, n² 27, 2014, 179 p., p. 21-31.

35. Paolo Portoghesi, "Rome et l'architecture organique », préface de Gilbert Picard, Empire romain, Fribourg, Office du Livre, 1965, p. 3-6.

36. Paolo Portoghesi, Le Inibizioni dell'architettura moderna, op. cit., p. 44.

37. Ibid., p. 67. Trad. fr. de l'auteur.

38. Luca Ribichini, "Il disegno di progetto: intervista a Paolo Portoghesi », Disegnare, idee, immagini, $n^{\circ}$ 9/10, décembre 1994 - juin 1995, p. 85-94.

39. Paolo Portoghesi, Le Inibizioni..., op. cit., p. 76. Trad. fr. de l'auteur.

40. La bibliographie de Paolo Portoghesi établie par Giovanna Massobrio et Maria Ercadi en 2001 recensait deux cent quarante-deux publications sur la mosquée de Rome dont trente-quatre à l'étranger et seulement treize pour la casa Baldi.

\section{RÉSUMÉS}

Paolo Portoghesi est une figure originale dans le panorama des historiens de l'architecture en Italie, notamment par son utilisation de la photographie comme outil d'analyse, lien entre l'histoire et le projet. Il lui consacre une place importante dans sa monographie sur Guarino Guarini (1956), dans Roma barocca (1966) ou encore dans ses monographies sur Bernardo Vittone (1966) et Borromini (1967). Cette utilisation a été remarquée (Pommer, Million et Wittkower), reprise par certains (Benevolo) et critiquée par d'autres (Zevi et Tafuri). Dans Roma un'altra città (1968), la photographie est au cœur du projet éditorial. Manfredo Tafuri évoque son travail de "photographie critique» et le risque de produire des images autonomes n'ayant plus de lien avec l'objet étudié : dans certains cas, l'abstraction prend le pas sur le caractère documentaire. La photographie de détail sert la comparaison formelle entre deux époques, référence à la pédagogie de Wölfflin. L'utilisation de contre-plongées met l'accent sur la géométrie des édifices et l'objectif "Fish-eye" accentue ces effets: on peut la considérer comme un équivalent photographique des perspectives plafonnantes d'Auguste Choisy. Ce travail de photographie critique s'inscrit dans la continuité de l'enseignement de l'école romaine d'histoire de l'architecture au sein de laquelle il s'affirme comme le meilleur représentant de la «critique opératoire ».

Paolo Portoghesi is a singular character in the scope of the historians of architecture in Italy noticeably through his use of photography as a tool to analyze the bond between history and 
projects. He gives it an important part in his monograph on Guarino Guarini (1956), Roma barocca (1966) or in his monographs about Bernardo Vittone (1966) and Borromini (1967). This use was pointed out (by Pommer, Million and Wittkower), embedded by some (Benevolo) and criticized by others (Zevi et Tafuri). In Roma un'altra città (1968), photography is at the heart of the editorial project. Manfredo Tafuri evokes his work on "critical photography » and the risk of taking autonomous pictures deprived of their link with the studied object: in some cases, abstraction takes advantage over the documentary side. Detailed photography is used as a formal comparison between two eras, in reference to Wölfflin's teaching. The use of low-angle shots puts emphasis on the geometry of the buildings as the "Fish eye» lens magnifies its effect: one could see it as a photographic equivalent of Auguste Choisy's " perspectives plafonnantes ». This work of critical photography takes shape in the continuity of the methodology of the roman school of historical architectural inside which he appears as the best ambassador of the «operational critic».

Paolo Portoghesi nimmt unter den Architekturhistorikern Italiens eine Sonderstellung ein, insbesondere durch seine Verwendung der Fotografie als Analyseinstrument, als Bindeglied zwischen Geschichte und Konzept. Er weist ihr eine bedeutende Rolle in seiner Monografie über Guarino Guarini (1956) zu, ebenso in Roma barocca (1966) oder auch in seinen Werken über Bernardo Vittone (1966) und Borromini (1967). Dieses Vorgehen wurde nicht nur rezipiert (Pommer, Million und Wittkower), sondern auch übernommen (Benevolo), jedoch bisweilen auch kritisiert (Zevi und Tafuri). In Roma un'altra città (1968) steht die Fotografie im Zentrum des editorischen Vorhabens. Manfredo Tafuri beschreibt das Vorgehen einer „kritischen Fotografie“ und das Risiko, autonome Bilder zu produzieren, die keine Verbindung mehr zum untersuchten objekt aufwiesen; in einigen Fällen überforme die Abstraktion den dokumentarischen Charakter der Aufnahme. Die Detailfotografie dient Portoghesi als Instrument eines formalen Vergleichs zweier Epochen und nimmt somit Bezug auf den pädagogischen Ansatz Wölfflins. Die Aufnahme aus der Froschperspektive akzentuiert die Geometrie des fotografierten Baus, wobei das „Fischaugen“Objektiv zusätzlich noch den Effekt verstärkt: So erscheinen Portoghesis Aufnahmen als fotografisches Pendant der Konstruktionszeichnungen eines Auguste Choisy. Die Arbeit einer „Photographie critique“ steht in der Tradition der römischen Schule der Architekturgeschichte, innerhalb derer sich Portoghesi als prominenter Vertreter einer „Verfahrenskritik“ erweist.

\section{AUTEUR}

\section{BENJAMIN CHAVARDES}

Benjamin Chavardes, après une formation de géomètre topographe, a étudié l'architecture à Montpellier et à Rome. Il est aujourd'hui architecte en Arles. En novembre 2014, il a soutenu une thèse en architecture sur l'œuvre de Paolo Portoghesi et les débats architecturaux de la seconde moitié $\mathrm{du} \mathrm{XX}^{\mathrm{e}}$ siècle, pour laquelle il a obtenu les félicitations du jury à l'unanimité et le label « doctorat européen ». Son jury de thèse était composé d'Alix Audurier-Cros, Jean-Louis Cohen, Claudia Conforti, Philippe Dufieux, Cristiana Mazzoni et Jean-François Pinchon. Allocataire du ministère de la Culture et de la Communication de 2010 à 2012, il a été boursier de l'école française de Rome à trois reprises, entre 2011 et 2012. Il a enseigné l'histoire de l'architecture et de la ville à l'École nationale supérieure d'architecture de Lyon, où il enseigne actuellement la théorie et la pratique de la conception architecturale et urbaine. Il a enseigné dans le séminaire « Patrimoine ancien, moderne et contemporain » de l'École nationale supérieure d'architecture de Paris Val-de-Seine, ainsi qu'à Montpellier, à Avignon et à la Politecnico de Turin. Enfin, il a été 
membre de jury dans les écoles d'architecture de Marseille et de Rouen. Adresse électronique :

benjamin.chavardes@hotmail.fr 